\title{
Relato de Experiência: o Uso de Simulações no Processo de Ensino-aprendizagem em Medicina
}

\author{
Report on an Experience with Simulations in the \\ Teaching/learning Process in Medicine
}

Cássia Regina Rodrigues Vargal Volia de Carvalho Almeida Carla Maria Ramos Germano ${ }^{I}$ Débora Gusmão Melo Silvana Gama Florêncio Chachál Bernardino Geraldo Alves Souto ${ }^{I}$ Bruno José Barcellos Fontanella Valéria Vernaschi Lima

\section{PALAVRAS-CHAVE}

- Educação médica.

- Aprendizagem baseada em problemas.

- Simulação de pacientes.

- Competência clínica.

\section{KEYWORDS}

- Medical education.

- Problem-based learning.

- Patient simulation.

- Clinical competence.

Recebido em: 28/12/2007

Reencaminhado em: 09/06/2008

Aprovado em: 23/07/2008

\section{RESUMO}

Este trabalho relata a experiência, construída ao longo de dois anos, com o uso de estações de simulação da prática profissional no processo de ensino-aprendizagem do curso de graduação em Medicina da Universidade Federal de São Carlos. Seu referencial pedagógico está ancorado na concepção construtivista da aprendizagem e parte da premissa de que aprender não é reproduzir a realidade, mas ser capaz de elaborar uma representação pessoal sobre esta e seus conteúdos. Assim, a partir da vivência de situações simuladas, os estudantes são estimulados a ressignificar seus conhecimentos construindo novos saberes. Na Unidade Educacional Estações de Simulação da Prática Profissional, o estudante se defronta com pacientes simulados e tem a oportunidade de aprender fazendo, errando e aprendendo com os próprios erros. Ao refletir sobre o erro, constrói seu aprendizado por meio da identificação de lacunas de conhecimento e fundamenta cognitivamente suas capacidades. Professores acompanham o desenvolvimento dos estudantes, atuando ora como avaliadores, ora como facilitadores, ora como consultores. Esta estratégia pedagógica pode ser um instrumento poderoso para o desenvolvimento de competência na área clínica.

\section{ABSTRACT}

This article reports on a two-year experience with Professional Practice Simulations (PPS) as a teaching/learning process in the undergraduate medical course at the Federal University in Sao Carlos, Brazil. This medical school's pedagogical approach is based on the constructivist conception of learning and the premise that learning is not reproducing reality, but being capable of elaborating a personal representation of reality and its contents. From this perspective, after experiencing simulated situations, students are encouraged to re-signify their previous scope of information, building new significant knowledge. In this educational unit (PPS), students interact with simulated patients and have the opportunity to learn by doing and analyzing their mistakes. While students reflect on their errors, they can identify the gaps in their knowledge and cognitively substantiate their skills. Teachers interact with students as evaluators, facilitators, or consultants. This pedagogical strategy can be a powerful tool for developing clinical skills. 


\section{INTRODUÇÃO}

O curso de Medicina da Universidade Federal de São Carlos (UFSCar) teve início em 2006, apresentando como diferencial a utilização da espiral construtivista como metodologia de ensino-aprendizagem e a integração teoria/prática ${ }^{1}$.

A metodologia construtivista busca substituir processos de memorização e de transferência fragmentada de informações do professor para o estudante. Parte da premissa de que aprender não é reproduzir a realidade, mas ser capaz de elaborar uma representação pessoal sobre esta e seus conteúdos ${ }^{1}$. Essa elaboração implica o desenvolvimento pessoal "na medida em que promove a atividade mental construtiva do aluno, responsável por transformá-lo em uma pessoa única, irrepetível, no contexto de um grupo social determinado"2 (p. 18).

Neste pressuposto teórico, o conhecimento é fruto de uma construção cuja constituição se dá nas relações socioculturais com seus significados e significantes e por força da própria ação humana. Essa construção é possível na medida em que ele tem a prática, a ação própria; e, também, na medida em que ele se apropria de teoria(s) suficientemente crítica(s) para dar conta das qualidades e dos limites de sua prática. Essas duas condições são absolutamente indispensáveis para o avanço do conhecimento e consequente ruptura com o senso comum na explicação do conhecimento. Assim, o conhecimento não é dado nem nos objetos (empirismo) nem na bagagem hereditária (apriorismo). O conhecimento é uma construção ${ }^{3}$.
Nessa perspectiva, o objetivo é, a partir da vivência de situações reais ou simuladas, ressignificar a aprendizagem construindo novos saberes voltados para a excelência profissional. Essas situações são vivenciadas pelos estudantes em três unidades educacionais: Unidade Educacional de Prática Profissional (UEPP), Unidade Educacional Eletiva (UEE) e Unidade Educacional de Simulação da Prática Profissional (UESP) ${ }^{1}$. A estrutura curricular dos dois primeiros anos do curso de Medicina da UFSCar é apresentada na Figura 1.

A UEPP é realizada em cenários reais da prática profissional. Os estudantes dos dois primeiros anos se vinculam às equipes de Saúde da Família e são acompanhados por professores no papel de preceptores e facilitadores. Os preceptores são profissionais de saúde vinculados aos serviços de saúde nos quais são desenvolvidas as atividades, e os facilitadores são docentes da UFSCar.

A UEE possibilita uma flexibilização do currículo, singularizada pelas necessidades dos estudantes com apoio de professores orientadores. É formada por atividades curriculares complementares que podem ser estruturadas como atividades de ensino, pesquisa e/ou extensão, tanto no cenário acadêmico como no do trabalho.

A UESPP é realizada em ambiente protegido na UFSCar. As atividades educacionais estão organizadas em duas atividades curriculares que utilizam disparadores simulados para o desenvolvimento do processo ensino-aprendizagem: situa-

FIGURA 1

Estrutura curricular dos dois primeiros anos do curso de Medicina da UFSCar.

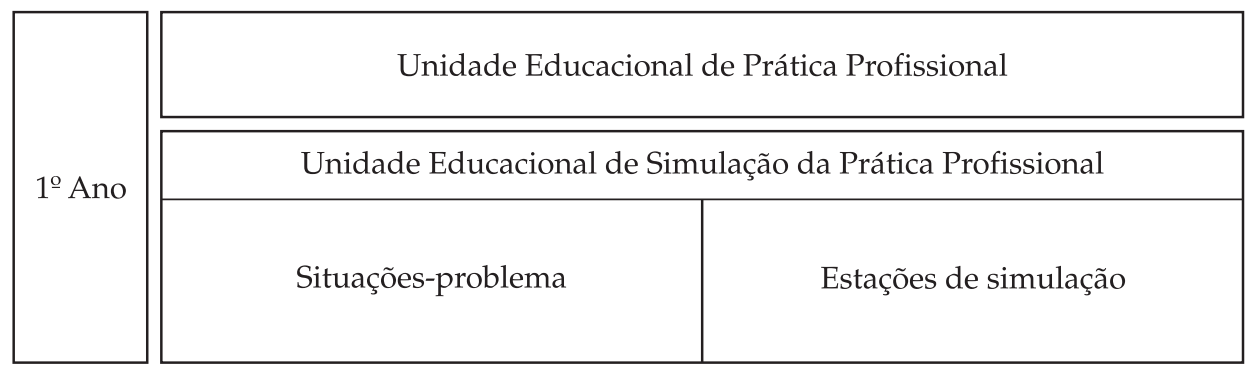

\begin{tabular}{|c|c|c|}
\hline \multirow{2}{*}{$2^{\circ}$ Ano } & \multicolumn{2}{|c|}{ Unidade Educacional de Prática Profissional } \\
\cline { 2 - 3 } & Unidade Educacional de Simulação da Prática Profissional \\
\hline & Situações-problema & Estações de simulação \\
\hline
\end{tabular}


ções-problema (SP) e Estações de Simulação da Prática Profissional (ESPP). Na SP, os elementos disparadores são situações-problema de papel, e na ESPP são situações simuladas. O objetivo da ESPP é construir com os estudantes a competência para identificar necessidades de saúde e elaborar planos de cuidado, por meio do desenvolvimento de capacidades cognitivas, afetivas e psicomotoras. Na ESPP, o conhecimento é construído a partir de situações programadas, representativas da realidade da prática médica, simuladas por pacientes-atores em ambiente protegido e controlado.

O processo de aprendizagem por meio de situações simuladas tem se mostrado um método útil e efetivo para avaliar desempenhos e habilidades clínicas, pois permite controle de fatores externos, padronização dos problemas apresentados pelos pacientes e feedback positivo para os alunos, aumentando o autoconhecimento e a confiança destes ${ }^{4,5}$. Dá oportunidade, ainda, para que a aprendizagem clínica seja centrada no paciente, garantindo melhor relacionamento interpessoal, resolução de problemas e análise e síntese das informações clínicas, mesmo sem a utilização de pacientes reais ${ }^{4,6}$.

O desenvolvimento dessa atividade é fundamentado no conceito de aprendizagem significativa concebido pelo psicólogo norte-americano David Paul Ausubel. Segundo Ausubel, para que uma nova informação faça sentido e seja apreendida pelo estudante, ela precisa se ancorar em conceitos relevantes previamente existentes na estrutura do aluno. São necessárias, portanto, duas condições para uma aprendizagem significativa: o aluno precisa ter uma disposição para aprender, e o conteúdo a ser aprendido tem que ser potencialmente significativo, ou seja, tem que ser lógica e psicologicamente significativo. O significado lógico depende somente da natureza do conteúdo, mas o significado psicológico é uma experiência que cada indivíduo tem. Quanto mais relevante para a vida do estudante for o conhecimento adquirido, mais capacidades podem ser desenvolvidas de forma efetiva no processo de aprendizagem. Cada estudante faz uma filtragem dos conteúdos que têm significado ou não para si próprio ${ }^{7}$.

Currículos que incorporam métodos de aprendizagem interativa, como as simulações, parecem ser mais bem-sucedidos do que aqueles que utilizam exclusivamente métodos tradicionais de ensino ${ }^{8,9,10}$. Além disso, a participação de atores interpretando pacientes é bem aceita por estudantes ${ }^{11,12}$, pois os atores também podem avaliar o desempenho do estudante, colaborando para enriquecer o processo de ensino-aprendizagem ${ }^{13}$.

A oportunidade de experimentar as novas habilidades a serem desenvolvidas, a diversificação dos cenários de aprendizagem e a possibilidade de refazer uma simulação são outras vantagens dessa estratégia.
A literatura mostra que os estudantes que têm contato o mais cedo possível com a prática simulada conseguem adquirir um arcabouço sólido de habilidades fundamentais em semiotécnica e comunicação, por se tratar de uma aprendizagem mais realista e significativa ${ }^{14}$

Essa estratégia pedagógica, entretanto, requer um planejamento cuidadoso e um custo que envolve, inclusive, capacitação de docentes e atores. Insere-se no modelo educacional uma complexidade que constitui o principal problema das simulações da prática. Por isso, o objetivo deste relato reflexivo é compartilhar a experiência do curso de Medicina da UFSCar, construída ao longo de dois anos, com o uso de estações de simulação da prática profissional no processo de ensino-aprendizagem.

\section{O funcionamento da ESPP}

O modelo esquemático de funcionamento da ESPP pode ser acompanhado na Figura 2.

No período de planejamento, os professores delimitam as competências a serem desenvolvidas em cada ciclo do curso, segundo o projeto pedagógico. A partir da competência esperada, é confeccionada uma matriz de intencionalidade que orienta a construção das estações, incluindo os roteiros de cada simulação. São as áreas de competência preestabelecidas no desenvolvimento esperado para cada ciclo, portanto, que direcionam a elaboração das situações que serão simuladas.

Ao construírem os roteiros das simulações, os professores planejam os contextos que serão abordados, considerando o que há de prevalente na prática profissional, segundo o perfil de competência desejado. As situações são criadas a partir da experiência médica dos professores e da vivência dos estudantes na UEPP.

Nos dois primeiros anos do curso, os cenários de aprendizagem nos quais ocorrem as simulações se baseiam no ambiente domiciliar e nas Unidades de Saúde da Família onde os estudantes desenvolvem a UEPP. O roteiro da cada simulação contém a ementa, o contexto, o papel a ser interpretado pelo ator e a tarefa daquele dia, tudo redigido de forma clara e coloquial. $\mathrm{O}$ ator é orientado para o papel que interpretará, o contexto apresenta o cenário simulado, e a tarefa define a atividade que será solicitada ao estudante.

As atividades com os estudantes são distribuídas em ciclos de quatro semanas de duração. Cada ciclo é composto por três momentos, um por semana. Como os estudantes trabalham em duplas, o primeiro momento, denominado avaliação formativa, é repetido nas duas primeiras semanas. Isso permite duas participações a ambos: uma simulando, e outra observando a simulação do colega. Os dois momentos seguintes, de 
FIGURA 2

Modelo esquemático do funcionamento da ESPP

Perfil de competência segundo o Projeto Pedagógico do Curso

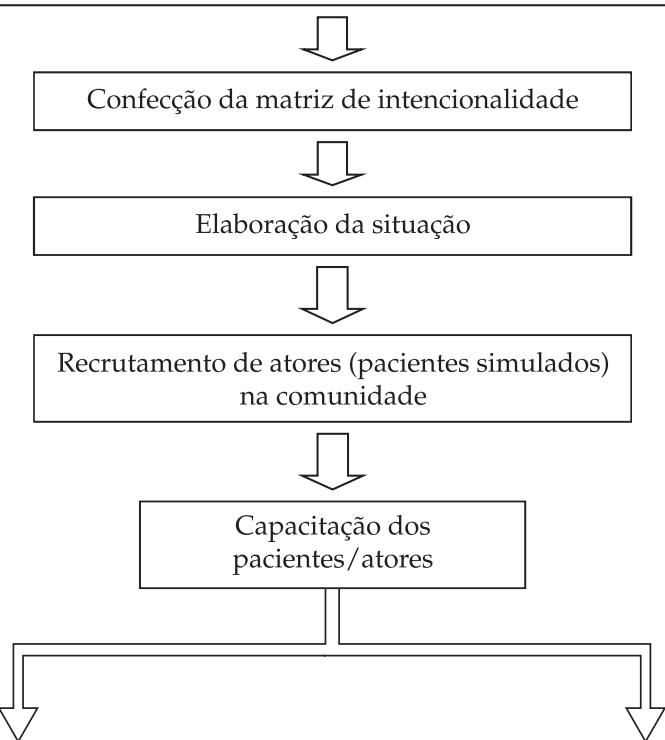

Momento 1A

Estudante $Y$ simula

Estudante $X$ observa e avalia

Professor observa e avalia

Paciente simulado atua e avalia

\section{Momento 1B}

Estudante $X$ simula

Estudante $\mathrm{Y}$ observa e avalia

Professor observa e avalia

Paciente simulado atua e avalia

Momento 1C

Estudante $Z$ simula

Estudante W observa e avalia

Professor observa e avalia

Paciente simulado atua e avalia

Momento 1D

Estudante W simula

Estudante Z observa e avalia

Professor observa e avalia

Paciente simulado atua e avalia

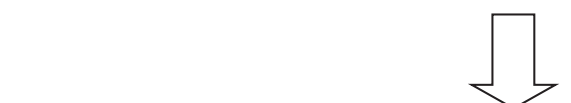

\section{Momento 2: Síntese Provisória}

Estudantes: X, Y, Z, W

Disparador: histórias clínicas, histórias de vida, narrativas sobre as avaliações do ator, do colega e/ou do professor

Tarefa: brain-storming, identificação de hiatos e fronteiras de conhecimento e formulação de questões de aprendizagem

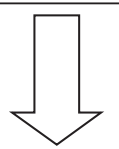

Momento 3: Nova Síntese

Estudantes: X, Y, Z, W

Tarefa: discussão do resultado das pesquisas bibliográficas

Professor fornece consultoria sobre pontos que o grupo solicita.

Eventual exercício de técnicas de semiologia e/ou de procedimentos 
acompanhamento, são chamados, respectivamente, de Síntese Provisória e Nova Síntese.

Essa forma de organização dá oportunidade aos estudantes de realizarem seis simulações na primeira série do curso, e cinco na segunda série.

Em cada simulação, o estudante se defronta com pacientes simulados em diferentes contextos e realiza sua tarefa em 40 minutos. O outro estudante da dupla permanece como observador crítico, sem interferir na ação do colega. O professor, nesse momento, atua como observador e avaliador.

Nesse ambiente protegido, o estudante tem oportunidade de aprender fazendo, errando e aprendendo com os próprios erros. Ao refletir sobre o erro, constrói sua aprendizagem por meio da identificação de lacunas em seu conhecimento e fundamenta cognitivamente suas habilidades.

Concluída a simulação, o estudante inicia o processo de avaliação, expressando suas considerações em relação ao próprio desempenho. Em seguida, o ator expressa seus sentimentos, como personagem, acerca do trabalho do estudante e deixa o ambiente. A partir daí, o professor solicita que o estudante fundamente as ações executadas, instigando o estudante a refletir sobre seu desempenho e sobre as ações que poderia ter desenvolvido, no âmbito cognitivo, atitudinal ou psicomotor. Nessa reflexão, o estudante expõe seu conhecimento prévio e respectivas fontes. O objetivo dessa busca é compreender de modo ampliado o desempenho do estudante. Nesse instante, o estudante que simulou é avaliado por seu colega, que, até então, se posicionava como observador. Por último, o professor avalia criticamente o trabalho desse estudante, salientando suas excelências e deficiências. Ao final, todos se autoavaliam, avaliam o desempenho do professor e o processo de trabalho daquele dia.

Na semana seguinte, os estudantes invertem seus papéis, mantendo-se a mesma dupla de colegas. Quem antes simulou se torna observador, e quem antes observou simulará. Assim, repete-se a mesma dinâmica de modo a assegurar a ambos a oportunidade de simular e observar a simulação do outro, como exposto anteriormente. O roteiro que o ator utiliza nesta segunda simulação é diferente do primeiro, embora tenha a mesma ementa e aborde os mesmos conteúdos, pois ambos têm matrizes de intencionalidade equivalentes.

Como um disparador de reflexão, os estudantes redigem uma narrativa da experiência ou uma história clínica do paciente representado pelo ator. Esse material será utilizado no próximo momento.

O acompanhamento, que ocorre na terceira semana do ciclo, chama-se Síntese Provisória. Reúnem-se o professor, agora no papel de facilitador, e os quatro estudantes para refletirem sobre as narrativas, histórias clínicas, avaliações ou fundamentações oriundas das simulações ocorridas nas duas semanas anteriores. Essa reflexão evidencia aos estudantes alguns hiatos e fronteiras de conhecimento que propiciam a formulação de questões de aprendizagem. Após levantarem tais questões, os presentes fazem uma síntese do que foi discutido no encontro e uma avaliação do dia de trabalho.

A Nova Síntese é realizada na quarta semana. Os estudantes trazem os resultados das pesquisas bibliográficas realizadas em estudos autodirigidos, individuais, em resposta às questões de aprendizagem levantadas durante a Síntese Provisória. Nessa oportunidade, os estudantes podem exercitar algumas habilidades psicomotoras, tendo o professor como facilitador e consultor ao mesmo tempo. Ao final, todos avaliam o dia de trabalho.

\section{O processo de avaliação do estudante na ESPP}

A avaliação de desempenho do estudante é realizada por meio de formulários de avaliação preenchidos semestralmente, cujo conteúdo e critérios de suficiência se apoiam nos referenciais de competência estabelecidos para a fase de desenvolvimento. Essa avaliação acaba por representar a síntese das avaliações dos estudantes realizadas pelos professores ao final de cada estação de simulação.

A avaliação estruturada do desempenho profissional do estudante é realizada ao término de cada série, pelos professores da UEPP e ESPP, por meio de simulações que reproduzem a vivência na ESPP. Esta avaliação é baseada em critérios. Os professores da unidade educacional, em conjunto, definem os desempenhos considerados satisfatórios a partir dos critérios estabelecidos como referência, e o avaliador descreve e qualifica o que observou. Na primeira série, a avaliação é formativa, enquanto na segunda a avaliação é somativa, implicando a progressão do estudante no currículo.

\section{O processo de avaliação da Unidade Educacional}

As avaliações escritas que os estudantes fazem dos professores e da Unidade são material de análise para um grupo de professores (grupo de avaliação), que faz análises qualitativas e quantitativas deste material e fornece um retorno aos professores da unidade educacional.

\section{DISCUSSÃO}

As simulações são utilizadas como estratégia de ensinoaprendizagem e de avaliação há cerca de 40 anos em diversos cenários educacionais. Após a introdução de pacientes simulados na educação médica, o desempenho baseado na avaliação de padrões objetivos tornou-se possível e seguro ${ }^{15}$. Atualmente, diversos instrumentos de avaliação utilizam pacientes simula- 
dos, como ocorre no Exame Clínico Estruturado e Objetivo (Objective Structured Clinical Examination - Osce) e no Accreditation Council of Graduate Medical Education and American Board of Medical Specialties ${ }^{16}$. No Canadá, o Conselho Médico (Medical Council) tem usado pacientes simulados no exame (avaliação) de licença nacional desde 1992, e o Conselho Nacional de Examinadores Médicos dos Estados Unidos (National Board of Medical Examiners) pretende incorporar essa estratégia na segunda fase do Exame de Licença ${ }^{17}$. A Escola Médica de Maastricht, na Holanda, usa pacientes simulados no ensino de habilidades de comunicação e do exame clínico há mais de duas décadas e considera as simulações uma importante ferramenta educacional ${ }^{17}$.

No ambiente protegido da simulação, o estudante tem a oportunidade de aprender fazendo, errando e aprendendo com os próprios erros. O erro é considerado matéria-prima para o desenvolvimento da consciência crítica. Reconhecendo as lacunas de seu conhecimento, o estudante tem a oportunidade de desenvolver novas fundamentações cognitivas e aprimorar suas capacidades de intervenção ${ }^{18}$.

Uma importante condição para que a simulação seja bemsucedida é realizar um planejamento cuidadoso. Os desempenhos esperados precisam estar bem definidos em cada ciclo educacional e série do curso, para nortearem a elaboração de situações simuladas adequadas. A construção da situação deve ser orientada por matrizes de intencionalidade que determinam os objetivos e o foco de aprendizagem a serem alcançados pelo estudante em cada simulação.

Na ESPP, os desempenhos esperados pelos estudantes na área de competência de saúde estão relacionados à identificação de necessidades de saúde, por meio da realização da história clínica e exame clínico, culminando com a elaboração de um plano terapêutico compatível. Esses desempenhos esperados vão evoluindo em grau de dificuldade e aprofundamento, respeitando a fase de aquisição de conhecimentos e o grau de autonomia dos estudantes, no sentido da construção do conceito de clínica ampliada.

O texto que descreve a simulação contém a ementa, o contexto e o roteiro, que é dirigido ao ator/paciente. Precisa ser redigido de forma clara, em linguagem popular, evitando-se termos técnicos. Deve conter o maior número possível de informações sobre a história da família e da vida dos indivíduos apresentados. A história precisa ter elementos suficientes para compor o perfil e o tipo de postura que o paciente/ator deve assumir naquela situação. $\mathrm{O}$ ator precisa ser previamente capacitado para o desenvolvimento da atividade. Por meio da história detalhada do personagem e de seu ambiente psicossociocultural, o ator obtém o máximo de informações a fim de ter domínio do papel que irá desempenhar. O contexto deve explicitar o cenário onde se desenvolve a simulação e trazer a atividade que será desenvolvida pelo estudante.

Em dois anos de experiência com este formato de organização das situações simuladas, as maiores dificuldades de operacionalização do processo se deveram, principalmente, ao custo e ao trabalho envolvidos para manter uma equipe adequada de pacientes simulados, que necessita ser capacitada e renovada continuamente.

Todo esse processo tem por objetivo propiciar ao estudante a construção de competência clínica e a vivência de uma prática reflexiva. Define-se competência como "um sistema de conhecimentos, relativos a conceitos e procedimentos, organizados em esquema operativos, que permitem, com respeito a uma gama de situações, a identificação de uma tarefa-problema, bem como a sua solução, por meio de uma ação eficaz"19 (p. 35). Essa definição abre caminho à concepção de um sujeito provido de um equipamento cognitivo complexo e que não se sujeita à sedimentação de certas reações condicionais. $\mathrm{O}$ que efetivamente introduz essa nova figura da competência como ação funcional não é apenas um sujeito epistêmico, ou cognitivo, mas também um sujeito na perspectiva de pessoa. A competência coloca em cena a intenção daquele que a possui, na medida em que ela é a faculdade de organizar os movimentos elementares visando a uma ação socialmente identificável. Não há competência sem objetivo. O exercício de uma competência, mesmo quando modesta, é um projeto ${ }^{20}$. Uma prática reflexiva pressupõe uma postura, uma forma de identidade, um habitus. Sua realidade não é medida por discursos ou por intenções, mas pelo lugar, pela natureza e pelas consequências da reflexão no exercício cotidiano da profissão ${ }^{21}$.

Na construção da competência profissional, a teoria construtivista introduz a perspectiva da imagem positiva do erro cometido pelo indivíduo, autor da ação, como mais fecundo e produtivo do que um acerto imediato. $\mathrm{O}$ indivíduo é entendido como ser ativo que paulatinamente seleciona melhores estratégias de ação que o levem a alcançar êxito em alguma tarefa proposta, em algum desafio que se lhe apresente. Portanto, a formação de bons profissionais tem a ver, acima de tudo, com a formação de pessoas capazes de evoluir, de aprender de acordo com a experiência, refletindo sobre o que gostariam de fazer, sobre o que realmente fizeram e sobre os resultados de tudo isso ${ }^{21}$.

\section{CONSIDERAÇÕES FINAIS}

Em suma, um profissional reflexivo só poderá ser formado por meio de uma prática reflexiva graças a essa fórmula paradoxal apreciada por Meirieu ${ }^{22}$ : aprender fazendo o que não se sabe fazer. Esse é o modelo profissional que buscamos formar no curso de Medicina da UFSCar. As avaliações, que 
serão feitas ao longo do curso, poderão responder de modo mais específico e com indicadores de impacto na aprendizagem e no grau de efetividade dessa estratégia educacional.

\section{REFERÊNCIAS}

1. Universidade Federal de São Carlos. Centro de Ciências Biológicas e da Saúde. Coordenação do Curso de Medicina. Caderno do Curso de Medicina. São Carlos: USFCar; 2006.

2. Solé I, Coll C. Os professores e a concepção construtivista. In: Coll C et al, orgs. O Construtivismo na sala de aula. São Paulo: Ática; 2001.

3. Becker F. Modelos pedagógicos e modelos epistemológicas. Educação e Realidade. 1994;19(1):89-96.

4. Howley LD, Martindale J. The efficacy of standardized patient feedback in clinical teaching: a mixed methods analysis. Med Educ Online. [on line]. 2004. 9(18):1-10. [Acesso em: 12 dez. 2007]. Disponível em: <http://med-ed-online. org/pdf/res00104.pdf>.

5. Curran VR, Butler R, Duke P, Eaton WH, Moffatt SM, Sherman GP, Pottle M. Evaluation of the usefulness of simulated clinical examination in family-medicine residency program. Med Teach 2007;29(4):406-7.

6. Smith SR, Cookson J, McKendree J, Harden RM. Patient-centred learning-back to the future. Med Teach. 2007;29(1):33-7.

7. Masini EFS, Moreira MA. A aprendizagem significativa: a teoria de David Ausubel. São Paulo: Centauro; 2001.

8. Barzansky B, Etzel SI. Educational programs in US medical schools, 2002-2003. JAMA. 290(9):1190-6.

9. Holm U, Aspegren K. Pedagogical methods and affect tolerance in medical students. Med Educ. 1999;33(1):14-28.

10. Howley LD. Performance Assessment in Medical Education: Where we've been and where we're going. Eval Health Prof. 2004; 27(3):285-303.

11. Quirk M, Letendre A. Teaching communication skills to first-year medical students. J Med Educ. 1986;61(7):603-5.

12. Rees C, Sheard C, McPherson A. Medical students' views and experiences of methods of teaching and learning communication skills. Patient Educ Couns. 2004;54(1):119-21.

13. Kurtz S. Teaching and learning communication in veterinary medicine. J Vet Med Educ. 2006; 33(1):11-19.

14. Dornan T, Littlewood S, Margolis SA, Scherpbier A, Spencer J, Ypinazar V. How can experience in: clinical and community settings contribute to early medical education? A BEME systematic review. Med Teach. 2006;28(1):3-18.

15. Barrows HS, Abrahamson S. The programmed patients: a technique for appraising student performance in clinical neurology. J Med Educ. 1964;39:802-5.
16. Accreditation Council of Graduate Medical Education. American Board of Medical Specialties. Toolbox of Assessment Methods. A product of the joint initiative of the ACGME. Outcome Project of the ACGME and the ABMS. [on line]. 2000; 1-25. [Acesso em: 12 dez. 2007]; Disponível em: <http:/ /www.acgme.org/Outcome/assess/Toolbox.pdf>.

17. Wind LA, van Dalen J, Muijtjens AMM, Rethans JJ. Assessing simulated patients in an educational setting: the MaSP (Maastricht Assessment of Simulated Patients). Med Educ. 2004;38(1):39-44.

18. Romão JE. Avaliação dialógica: desafios e perspectivas. São Paul;, Cortez, 1998.

19. Gillet apud Rey B. As competências transversais em questão. Porto Alegre: Artmed; 2002.

20. Rey B. As competências transversais em questão. Porto Alegre: Artmed; 2002

21. Perrenoud P. A prática reflexiva no ofício de professor: profissionalização e razão pedagógica. Porto Alegre: Artmed; 2002.

22. Meirieu P. Aprender- sim, mas como? Porto Alegre: Artmed; 1998.

\section{CONTRIBUIÇÃO DOS AUTORES}

Cássia Regina Rodrigues Varga: delineamento teórico e desenvolvimento da experiência educacional, redação do artigo. Volia de Carvalho Almeida: delineamento teórico e desenvolvimento da experiência educacional, redação do artigo. Carla Maria Ramos Germano: delineamento teórico e desenvolvimento da experiência educacional, redação do artigo. Débora Gusmão Melo: delineamento teórico e desenvolvimento da experiência educacional, redação do artigo. Silvana Gama Florêncio Chachá: delineamento teórico e desenvolvimento da experiência educacional, redação do artigo. Bernardino Geraldo Alves Souto: delineamento teórico e desenvolvimento da experiência educacional, redação do artigo. Bruno José Barcellos Fontanella: delineamento teórico e desenvolvimento da experiência educacional, redação do artigo. Valéria Vernaschi Lima: delineamento teórico da experiência educacional.

\section{CONFLITO DE INTERESSES}

Declarou não haver

\section{ENDEREÇO PARA CORRESPONDÊNCIA}

Cassia Regina R. Varga

Campus da UFSCar, Departamento de Medicina

Rod. Washington Luis (SP-310) - Km 235

CEP.: 13565-905 - São Carlos - SP

E-mails: cassiavarga@terra.com.br 